\title{
Unitary Precoding for Integer-Forcing MIMO Linear Receivers
}

\author{
Amin Sakzad and Emanuele Viterbo \\ Department of Electrical \& Computer Systems \\ Monash University, Australia \\ amin.sakzad, emanuele.viterbo@monash.edu
}

\begin{abstract}
A flat fading point-to-point multiple-antenna channel is considered where the channel state information is known at both transmitter and receiver. At the transmitter side, we use a lattice encoder to map information symbols to lattice codewords. The lattice coded layers are then precoded using unitary matrices satisfying non-vanishing minimum product distance. At the receiver side, an integer-forcing linear receiver is employed. This scheme is called 'unitary precoded integer-forcing'. We show that by applying the proposed precoding technique full-diversity can be achieved. We then verify this result by conducting computer simulations in a $2 \times 2$ and $4 \times 4$ multiple-input multiple-output (MIMO) channel using full-diversity algebraic rotation precoder matrices.
\end{abstract}

Index Terms-Integer-Forcing, unitary precoding, lattice codes, full-diversity.

\section{INTRODUCTION}

Multiple-input multiple-output (MIMO) channels with multiple antennas appeared in the early 1990's as the key technology to achieve high spectral efficiencies in wireless channels. On one hand, optimal maximum likelihood (ML) detectors such as sphere decoder [1] have high computational complexity. On the other hand, there are well-known linear receivers [2] including zero-forcing (ZF) and minimum mean square estimation (MMSE) linear receivers, which trade-off error performance for reduced computational complexity. In these schemes, the channel coefficient matrix is forced to an identity matrix.

The integer-forcing (IF) linear receiver has been recently proposed in [3] to obtain higher rates in MIMO channels with reduced decoding complexity. In this framework, the transmitter employs a lattice coding scheme, and sends independent lattice codewords simultaneously across different layers. Identical lattice codes carved from lattices are used as codebooks for each transmit antenna and hence, at the destination, each receive antenna decodes an integer linear combination of transmitted lattice codewords. The decoded point is another lattice point because any integer linear combination of lattice points is another lattice point. This makes detecting information symbols easier by only solving a system

This work was performed at the Monash Software Defined Telecommunications (SDT) Lab. A. Sakzad was supported by the Australian Research Council under Discovery grants ARC DP 130100103 and E. Viterbo was supported by NPRP grant NPRP5-597-2-241 from the Qatar National Research Fund (a member of Qatar Foundation). of linear equations with integer coefficients in each codeword transmission. Since the matrix of coefficients for this system needs to be a non-singular integer matrix, this scheme is called integer-forcing. It is also shown in [3], [4] that integer-forcing linear receiver provides a full receive diversity order and a full multiplexing gain. The problem of finding design parameters for IF receivers using lattice reduction algorithms is addressed in [4], [5]. Therefore, there is one advantage in using IF linear receiver over ML decoders such as sphere decoder. For the IF receiver, the lattice reduction algorithm is performed only at the beginning of each quasi-static interval for which the channel is assumed to remain constant for a long time so that many codewords are transmitted. Subsequently, after each codeword transmission, only a system of linear equations has to be solved to recover an estimate of that codeword. Instead, for the ML decoding, a sphere decoder algorithm should be performed at every codeword use within a quasi-static interval. Hence, the complexity of the proposed IF receiver is lower than the ML decoder for slow fading channels.

Space-Time Block-Codes (STBC) are designed to maximize both diversity and coding gain in MIMO channels where an optimal decoder is available at the receiver. The design parameters for these codes are known to be the rank and the non-vanishing determinant criteria [6], [7]. In addition, families of full-diversity space-time codes for which linear receivers ( $\mathrm{ZF}$ and MMSE only) are used at the receiver, were fully investigated and constructed in [8], [9], [10], [11], [12], [13]. The MIMO linear precoding techniques such as regularized ZF [14] are alternative approaches that can provide full diversity in conjunction with linear receivers like MMSE, see [15] and references therein. In particular, using perfect STBCs like Golden Codes, the authors of [16] have pointed out that under an IF decoder, a constant gap to capacity is attainable. A perfect STBC [17] code is a linear dispersion space-time code over a Quadrature-Amplitude Modulation (QAM) constellation, which is full-rate, satisfies non-vanishing determinant property, and its generator matrix is a unitary matrix. We aim at relaxing the first two constraints of perfect STBCs and keeping the last one only. We analyze the diversity order of a lattice space-time encoding scheme, which is precoded by a unitary matrix for IF linear receiver. We approach the problem of precoder design by using the singular value decomposition (SVD) to diagonalize the channel, and 
then implemen the optimal solution for each of the resulting parallel scalar channels. Our optimization criterion is based on maximizing the minimum distance of the input lattice, similar to the approach of [18]. We note that differently from [18], our precoder matrices are unitary and our decoder is the IF linear receiver and not the ML receiver. Other work ([19], [20], [21], and [22] and references therein) focus on a different design criterion based on maximizing the mutual information.

In this paper, using channel state information at both transmitter and receiver (CSIT and CSIR, respectively), we propose a unitary precoding scheme for $n \times n$ MIMO channels, where the detector at the receiver is IF. In particular, the well-known SVD along with a unitary precoding matrix, which satisfies the non-vanishing minimum product distance criterion are used together to produce unitary precoding matrices. We define this scheme as unitary precoded integer-forcing (UPIF). It is shown analytically that full (transmit and receive) diversity can be achieved by employing the introduced UPIF. The computer simulations were conducted using a well-known full-diversity algebraic rotation [23] and presented to support the derived theoretical results. For example, it is shown that if a $2 \times 2$ full-diversity algebraic rotation matrix given in [24] is used over a UPIF scheme, it can perform as close as $1 \mathrm{~dB}$ away from ML decoder for the same code.

The rest of the paper is organized as follows: In Section II we formulate our problem and introduce unitary-precoded integer-forcing scheme. Diversity analysis of the proposed scheme is provided in Section III. We finally conduct simulations to verify the correctness of our theoretical results in Section IV. We conclude with some comments and future directions.

Notation. Capital letters are used for functions, and capital calligraphic letters for sets. The superscript ${ }^{h}$ denotes Hermitian transposition. Let $\mathcal{G}$ and $\mathcal{G}^{\prime}$ be a group and its subgroup respectively, then $\mathcal{G} / \mathcal{G}^{\prime}$ denotes the quotient group. The sets $\mathbb{Z}, \mathbb{C}, \mathbb{R}$, and $\mathbb{Z}[i]$ denote the ring of rational integers, the field of complex numbers, the field of real numbers, and the ring of Gaussian integers, respectively, where $i^{2}=-1$. We further denote $\mathbb{C} \backslash\{0\}$ by $\mathbb{C}^{*}$. The operations $\Re(\cdot)$ and $\Im(\cdot)$ denote the real and imaginary parts of a complex number. We let $|z|$ and $\arg (z)$ denote the modulus and the unique phase of the complex number $z$, respectively. The notation $\|\mathbf{v}\|$ stands for the Euclidean norm of a vector $\mathbf{v} \in \mathbb{C}^{n}$. Finally, an $k \times k$ matrix $\mathbf{X}=\left[\mathbf{x}_{1}^{T}, \ldots, \mathbf{x}_{k}^{T}\right]^{T}$ is formed by stacking the $k$-dimensional row vectors $\mathbf{x}_{1}, \ldots, \mathbf{x}_{k}$, and $\mathbf{I}_{k}$ denote the $k \times k$ identity matrix. Finally, let $\mathbf{v}$ be a vector, then its $j$-th entry is represented by $[\mathbf{v}]_{j}$.

\section{System Model}

We first summarize the notion of complex lattices [25] which are essential for the rest of the paper. A $k$-dimensional lattice $\Lambda$ with a basis set $\left\{\boldsymbol{\ell}_{1}, \ldots, \boldsymbol{\ell}_{k}\right\} \subseteq \mathbb{C}^{d}$ is the set of all Gaussian integer linear combinations of basis vectors. Let $\mathbf{L}$ be a matrix with $\boldsymbol{\ell}_{m}$ as its rows, then $\mathbf{L}$ is called the generator matrix of the lattice $\Lambda_{\mathbf{L}}$. Throughout the paper, we only consider full rank lattices where $d=k$. For example $\mathbb{Z}[i]^{d}$ is a lattice with standard bases. For an $d$-dimensional lattice $\Lambda_{\mathbf{L}}$, we define the $m$-th successive minima, for $1 \leq m \leq d$ as

$$
\epsilon_{m}\left(\Lambda_{\mathbf{L}}\right) \triangleq \inf \left\{r: \operatorname{dim}\left(\Lambda_{\mathbf{L}} \cap \mathcal{N}_{r}(\mathbf{0})\right) \geq m\right\},
$$

where

$$
\mathcal{N}_{r}(\mathbf{0})=\left\{\mathbf{x} \in \mathbb{R}^{d}:\|\mathbf{x}\| \leq r\right\},
$$

and $\operatorname{dim}(\mathcal{V})$ denotes the dimension of subspace $\mathcal{V} \subseteq \mathbb{R}^{d}$. A $d$ dimensional lattice $\Lambda_{\mathbf{L}}$ is called full-diversity if for all disjoint $\mathbf{x}, \mathbf{y} \in \Lambda_{\mathbf{L}}$, the number of elements in $\left\{m:[\mathbf{x}]_{m} \neq[\mathbf{y}]_{m}\right\}$ be exactly $d$. The minimum product distance of a full-diversity lattice $\Lambda_{\mathbf{L}}$ is denoted by $d_{p, \min }\left(\Lambda_{\mathbf{L}}\right)$ and is defined by [23]:

$$
d_{p, \min }\left(\Lambda_{\mathbf{L}}\right) \triangleq \min _{\mathbf{0} \neq \mathbf{x} \in \Lambda_{\mathbf{L}}} \prod_{m}\left|[\mathbf{x}]_{m}\right|
$$

A subset $\Lambda^{\prime} \subseteq \Lambda$ is called a sublattice if $\Lambda^{\prime}$ is a lattice itself. Given a sublattice $\Lambda^{\prime}$, we define the quotient $\Lambda / \Lambda^{\prime}$ as a lattice code. This corresponds to a finite constellation of lattice points carved from the lattice $\Lambda$. The shape of such constellation is governed by the Voronoi region of the shaping lattice $\Lambda^{\prime}$ as explained in [25]. A common choice for the sublattice $\Lambda^{\prime}$ is $g \Lambda$ for some integer $g \in \mathbb{Z}$. For example, for $\Lambda=\mathbb{Z}[i]^{d}$ and $\Lambda^{\prime}=g \mathbb{Z}[i]^{d}$, we have $\Lambda / \Lambda^{\prime}=\mathbb{Z}_{g}[i]$ for which $\mathbb{Z}_{g}$ is the ring of integers modulo $g$. This is called hypercube shaping. For $g=2$ the cubic shaped lattice code $\mathbb{Z}_{2}[i]$ is simply a 4 -QAM constellation. For the rest of this paper we use $\Lambda=\mathbb{Z}[i]^{d}$ and $g$ a power of 2 .

We consider a quasi-statistic flat-fading $n \times n$ MIMO channel, where the channel state information is available at both transmitter and receiver. The channel matrix is denoted by $\overline{\mathbf{H}} \in \mathbb{C}^{n \times n}$, where the entries of $\overline{\mathbf{H}}$ are i.i.d. complex Gaussian random variables $\sim \mathcal{C N}(0,1)$. We use an $n$-layer lattice coding scheme, where the information transmitted across different antennas are independent. For $1 \leq m \leq n$, the $m$ th layer is equipped with a lattice encoder $E: \mathcal{R}^{k} \rightarrow \mathbb{C}^{n}$ which maps a message $\overline{\mathbf{s}}_{m} \in \mathcal{R}^{k}$ over the ring $\mathcal{R}$ into a lattice codeword $\overline{\mathbf{x}}_{m} \in \Lambda / \Lambda^{\prime} \subset \mathbb{C}^{n}$ in the complex space. The matrix $\overline{\mathbf{X}}=\left[\overline{\mathbf{x}}_{1}^{T}, \ldots, \overline{\mathbf{x}}_{n}^{T}\right]^{T}$ is actually a space-time codeword, where its rows are all lattice codewords. This lattice spacetime codeword will then be precoded using a unitary matrix. The precoding matrix $\overline{\mathbf{U}}$ can be derived using the components of the channel matrix $\overline{\mathbf{H}}$ and another unitary matrix $\overline{\mathbf{P}}$, to be optimized later. Together with $\overline{\mathbf{U}}$, the matrix $\overline{\mathbf{X}}$ forms a space-time codeword $\overline{\mathbf{U}} \overline{\mathbf{X}}$ to be sent through the channel.

Let $\overline{\mathbf{H}}=\overline{\mathbf{W}} \overline{\boldsymbol{\Sigma}} \overline{\mathbf{V}}^{h}$ be the singular value decomposition (SVD) of the channel matrix where $\overline{\mathbf{W}}, \overline{\mathbf{V}} \in \mathbb{C}^{n \times n}$ are two unitary matrices and $\overline{\boldsymbol{\Sigma}}$ is a diagonal matrix given by $\overline{\boldsymbol{\Sigma}}=\operatorname{diag}\left(\overline{\sigma_{1}}, \ldots, \overline{\sigma_{n}}\right)$ with $\overline{\sigma_{1}} \geq \cdots \geq \overline{\sigma_{n}}$ all in $\mathbb{R}$. A unitary precoder matrix

$$
\overline{\mathbf{U}}=\overline{\mathbf{V}} \overline{\mathbf{P}}
$$

is then employed at the transmitter where $\overline{\mathbf{P}} \in \mathbb{C}^{n \times n}$ is a unitary matrix that needs to be optimized. If $\overline{\mathbf{X}}=\left[\overline{\mathbf{x}}_{1}^{T}, \ldots, \overline{\mathbf{x}}_{n}^{T}\right]^{T}$ denotes the matrix of transmitted vectors, the received signal $\overline{\mathbf{Y}}$ is given by

$$
\overline{\mathbf{Y}}=\sqrt{\rho} \cdot \overline{\mathbf{H}} \overline{\mathbf{U}} \overline{\mathbf{X}}+\overline{\mathbf{Z}}
$$


where $\rho=\frac{\text { SNR }}{n}$ and SNR denotes the average signal-tonoise ratio at each receive antenna and the entries of $\overline{\mathbf{Z}}$ are i.i.d. distributed as $\mathcal{C N}(0,1)$. Using standard conversion from complex lattice to the equivalent real one, we have the real lattice generator matrix as:

$$
\mathbf{M}=\left(\begin{array}{cc}
\Re(\overline{\mathbf{M}}) & \Im(\overline{\mathbf{M}}) \\
-\Im(\overline{\mathbf{M}}) & \Re(\overline{\mathbf{M}})
\end{array}\right) .
$$

With the above transformation, (4) can be written as

$$
\mathbf{Y}=\sqrt{\rho} \cdot \mathbf{H U X}+\mathbf{Z},
$$

where all the matrices $\mathbf{Y}, \mathbf{H}, \mathbf{U}, \mathbf{X}$, and $\mathbf{Z}$ are in $\mathbb{R}^{2 n \times 2 n}$. A suitable block diagram is then as Fig. 1. Upon receiving $\mathbf{Y}$ at the destination, we multiply it by $\mathbf{W}^{h}$ to get $\mathbf{Y}^{\prime} \triangleq \mathbf{W}^{h} \mathbf{Y}$. Substituting $\mathbf{U}$ from (3) into (5) the channel can be modeled as:

$$
\mathbf{Y}^{\prime}=\sqrt{\rho} \cdot \mathbf{\Sigma} \mathbf{P} \mathbf{X}+\mathbf{Z}^{\prime}
$$

where $\mathbf{Z}^{\prime}=\mathbf{W}^{h} \mathbf{Z}$. Note that $\mathbf{Z}^{\prime}$ continues to be an i.i.d. complex Gaussian matrix with entries $\sim \mathcal{C N}(0,1)$ because $\mathbf{W}$ is unitary.

The goal of integer-forcing linear receiver is to project $\mathbf{\Sigma P}$ (by left multiplying it with a receiver filtering matrix $\mathbf{B}$ ) onto a non-singular integer matrix $\mathbf{A}$. In order to uniquely recover the information symbols, the matrix $\mathbf{A}$ must be invertible over the ring $\mathcal{R}$. Thus, we have

$$
\mathbf{Y}^{\prime \prime}=\mathbf{B} \mathbf{Y}^{\prime}=\sqrt{\rho} \cdot \mathbf{B} \mathbf{\Sigma} \mathbf{P} \mathbf{X}+\mathbf{B Z} .
$$

For the IF receiver [3] formulation, the model is given by

$$
\begin{aligned}
\mathbf{Y}^{\prime \prime} & =\sqrt{\rho} \cdot \mathbf{A X}+\underbrace{\sqrt{\rho} \cdot(\mathbf{B} \boldsymbol{\mathbf { }} \mathbf{P}-\mathbf{A}) \mathbf{X}}_{\text {quantization noise term }}+\mathbf{B} \mathbf{Z}^{\prime} \\
& \triangleq \sqrt{\rho} \cdot \mathbf{A X}+\mathbf{E},
\end{aligned}
$$

where $\sqrt{\rho} \cdot \mathbf{A X}$ is the desired signal component, and the effective noise is

$$
\sqrt{\rho} \cdot(\mathbf{B} \boldsymbol{\Sigma} \mathbf{P}-\mathbf{A}) \mathbf{X}+\mathbf{B Z}^{\prime} .
$$

We further denote the effective noise term along the $m$-th layer by $\mathbf{e}_{m}$. The average energy of the effective noise along the $m$-th row of $\mathbf{Y}^{\prime \prime}$ is defined as

$$
G\left(\mathbf{a}_{m}, \mathbf{b}_{m}\right) \triangleq \rho\left\|\mathbf{b}_{m} \boldsymbol{\Sigma} \mathbf{P}-\mathbf{a}_{m}\right\|^{2}+\left\|\mathbf{b}_{m}\right\|^{2},
$$

where $\mathbf{a}_{m}$ and $\mathbf{b}_{m}$ denote the $m$-th row of $\mathbf{A}$ and $\mathbf{B}$, respectively. Note that in order to increase the effective signalto-noise ratio for each layer, the term $G\left(\mathbf{a}_{m}, \mathbf{b}_{m}\right)$ has to be minimized for each $m$ by appropriately selecting the matrices $\mathbf{A}$ and $\mathbf{B}$. We refer to the above signal model as unitary precoded integer-forcing.

\section{Diversity ANALysis}

We first recall Woodbury identity [26], which is used in the rest of this Section. Let $\mathbf{U}$ and $\mathbf{V}$ be two $n \times n$ invertible matrices, then the following identity holds:

$$
\left(\mathbf{I}_{n}+\mathbf{U V}\right)^{-1}=\mathbf{I}_{n}-\mathbf{U}\left(\mathbf{I}_{n}+\mathbf{V U}\right)^{-1} \mathbf{V} .
$$

Note that the effective noise is not Gaussian distributed due to the quantization noise term. However, since the optimum value of $\mathbf{b}_{m}$ that minimizes (9) given $\mathbf{a}_{m}$ is [3]

$$
\begin{aligned}
\mathbf{b}_{m} & =\rho \cdot \mathbf{a}_{m} \boldsymbol{\Sigma} \mathbf{P}^{h}\left(\mathbf{I}_{2 n}+\rho \cdot \boldsymbol{\Sigma} \mathbf{P}(\boldsymbol{\Sigma} \mathbf{P})^{h}\right)^{-1} \\
& \triangleq \rho \cdot \mathbf{a}_{m}(\boldsymbol{\Sigma} \mathbf{P})^{h} \mathbf{S}^{-1}
\end{aligned}
$$

the quantization noise term along the $m$-th layer is then given by

$$
\begin{aligned}
G\left(\mathbf{a}_{m}, \mathbf{b}_{m}\right) & =\rho\left\|\mathbf{b}_{m} \boldsymbol{\Sigma} \mathbf{P}-\mathbf{a}_{m}\right\|^{2}+\left\|\mathbf{b}_{m}\right\|^{2} \\
& =\rho \cdot \mathbf{a}_{m}\left(\mathbf{I}_{2 n}-(\boldsymbol{\Sigma} \mathbf{P})^{h} \mathbf{S}^{-1} \boldsymbol{\Sigma} \mathbf{P}\right) \mathbf{a}_{m}^{h} \\
& =\rho \cdot \mathbf{a}_{m}\left(\mathbf{I}_{2 n}+\rho \cdot(\boldsymbol{\Sigma} \mathbf{P})^{h} \boldsymbol{\Sigma} \mathbf{P}\right)^{-1} \mathbf{a}_{m}^{h}(11) \\
& =\rho \cdot \mathbf{a}_{m} \mathbf{P}^{h}\left(\mathbf{I}_{2 n}+\rho \cdot \boldsymbol{\Sigma}^{h} \boldsymbol{\Sigma}\right)^{-1} \mathbf{P a}_{m}^{h} \\
& =\rho \cdot \mathbf{a}_{m} \mathbf{P}^{h} \mathbf{L} \mathbf{L}^{h} \mathbf{P} \mathbf{a}_{m}^{h} \\
& \triangleq \rho \cdot \mathbf{a}_{m} \mathbf{L}_{p} \mathbf{L}_{p}^{h} \mathbf{a}_{m}^{h}
\end{aligned}
$$

where (10) holds because of (9) in [4], (11) is true because of the Woodbury identity [26] for

$$
\mathbf{U}=\sqrt{\rho} \cdot(\boldsymbol{\Sigma} \mathbf{P})^{h}, \quad \mathbf{V}=\sqrt{\rho} \cdot \boldsymbol{\Sigma} \mathbf{P},
$$

and (12) uses the fact that $\left(\mathbf{I}_{2 n}+\rho \cdot \boldsymbol{\Sigma}^{h} \boldsymbol{\Sigma}\right)^{-1}$ is a positive definite matrix which has the Cholesky decomposition $\mathbf{L L}^{h}$.

We denote the probability of error for decoding the $m$-th layer in the infinite lattice $\mathbb{Z}$ by $P_{e}(m, \boldsymbol{\Sigma P}, \mathbb{Z})$. It follows that

$$
P_{e}(m, \boldsymbol{\Sigma P}, \mathbb{Z})=\operatorname{Pr}\left(\left|\mathbf{e}_{m}\right| \geq \frac{\sqrt{\rho}}{2}\right) .
$$

The following Theorem provides an upper bound on $P_{e}(m, \mathbf{\Sigma} \mathbf{P}, \mathbb{Z})$. Due to lack of space, we remove the proofs of the following two theorems and include them in the extended version of this paper.

Theorem 1: (Upper Bound on Probability of Error) The term $P_{e}(m, \mathbf{\Sigma} \mathbf{P})$, for all $1 \leq m \leq 2 n$, is upper bounded as

$$
P_{e}(m, \mathbf{\Sigma P}, \mathbb{Z}) \leq \exp \left(-c \epsilon_{2 n-m+1}^{2}\left(\Lambda_{\mathbf{L}_{p}^{-1}}\right)\right),
$$

where $c$ is some constant independent of $\rho$ and $\epsilon_{2 n-m+1}^{2}\left(\Lambda_{\mathbf{L}_{p}^{-1}}\right)$ is the $(2 n-m+1)$-th successive minima of the lattice with generator matrix $\mathbf{L}_{p}^{-1}$.

We are interested in $P_{e}(2 n, \boldsymbol{\Sigma} \mathbf{P}, \mathbb{Z})$ which is an upper bound for $P_{e}(m, \boldsymbol{\Sigma} \mathbf{P}, \mathbb{Z})$, for all $1 \leq m \leq 2 n$. We define the error probability for phase precoded integer forcing over $\mathbb{Z}$ as

$$
P_{e}(\boldsymbol{\Sigma} \mathbf{P}, \mathbb{Z}) \triangleq P_{e}(2 n, \boldsymbol{\Sigma} \mathbf{P}, \mathbb{Z}) .
$$

Based on (14), we have

$$
P_{e}(\boldsymbol{\Sigma} \mathbf{P}, \mathbb{Z}) \leq \exp \left(-c \epsilon_{1}^{2}\left(\Lambda_{\mathbf{L}_{p}^{-1}}\right)\right)
$$

Theorem 2: Let the precoding matrix $\mathbf{P}$ be such that $d_{p, \min }\left(\Lambda_{\mathbf{P}}\right) \neq 0$, then the achievable diversity of the unitary precoded integer-forcing is $(2 n)^{2}$.

According to the above theorem, if lattice codes along with a non-zero minimum product distance precoder are used at 


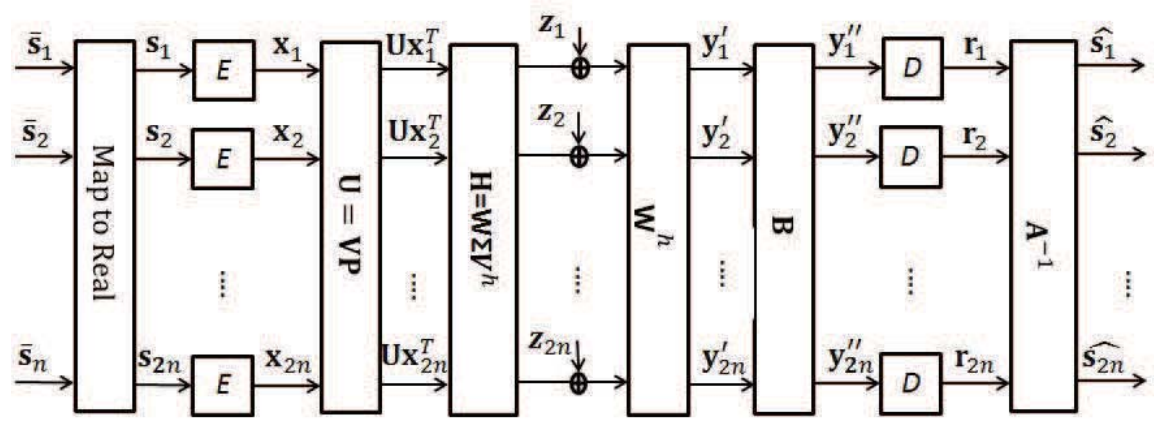

Fig. 1. Real Block diagram of unitary precoded integer-forcing.

the transmitter of a MIMO channel and integer-forcing is employed at the receiver, full (transmit and receive) diversity can be achieved. We now proceed to verify this result by conducting simulations.

\section{Simulation Results}

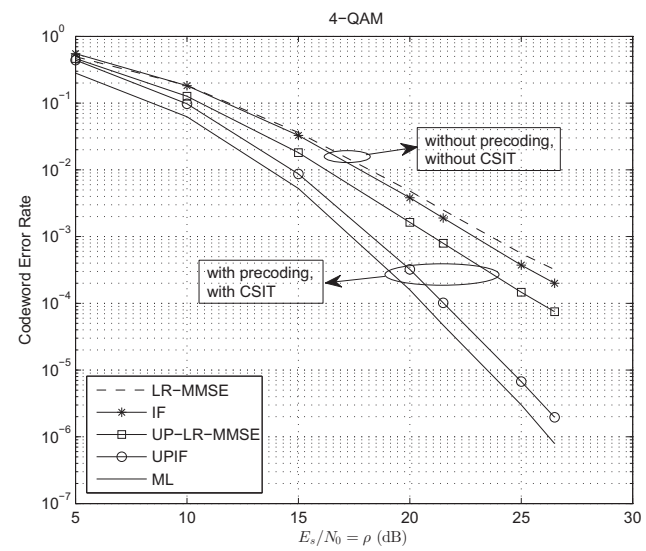

Fig. 2. Unitary Precoded IF in comparison with other schemes in a $2 \times 2$ MIMO Channel. The precoding matrix $\mathbf{P}_{2}$ as in (17) is employed.

We have conducted simulation for a $2 \times 2$ MIMO UPIF channel over a 4-QAM constellation. In this case, the matrices $\mathbf{A}$ and $\mathbf{B}$ for IF linear receiver were found using Algorithm2 presented in [4]. The full-diversity algebraic rotation [24] matrix $\mathbf{P}_{2}$ which has been employed for simulations is as follows:

$$
\mathbf{P}_{2}=\left(\begin{array}{cc}
-0.5257311121 & -0.8506508083 \\
-0.8506508083 & 0.5257311121
\end{array}\right),
$$

with $d_{p, \min }\left(\Lambda_{\mathbf{P}_{2}}\right)=.4472$. For reference purposes, we also presented IF with no precoding and no CSIT along with unitary precoded scheme decoded under ML detector. Fig. 2 shows the codeword error rate [4] curves versus signal-to-noise ratio $\rho$ of different mentioned schemes over a $2 \times 2$ MIMO channel. The change of slope from no precoding curve to unitary precoded schemes is obvious in Fig. 2. In particular, at codeword error rate $10^{-4}$ a gap of less than $1 \mathrm{~dB}$ is observed, which shows strong agreement between derived numerical results and provided theoretical achievements.

The same set of simulations were conducted for a $4 \times 4$ MIMO channel, where a full-diversity algebraic rotation [24] matrix $\mathbf{P}_{4}$ as in (19) with $d_{p, \min }\left(\Lambda_{\mathbf{P}_{4}}\right)=0.0371$ have been used. The codeword error rate [4] curves versus signal-to-noise ratio $\rho$ of the precoded schemes with different decoders were shown in Fig. 3. We calculated the matrices $\mathbf{A}$ and $\mathbf{B}$ for IF linear receiver with Algorithm1 along with HKZ lattice reduction presented in [4]. In particular, at codeword error rate $10^{-3}$ a gap of less than $2.5 \mathrm{~dB}$ away from ML performance is observed.

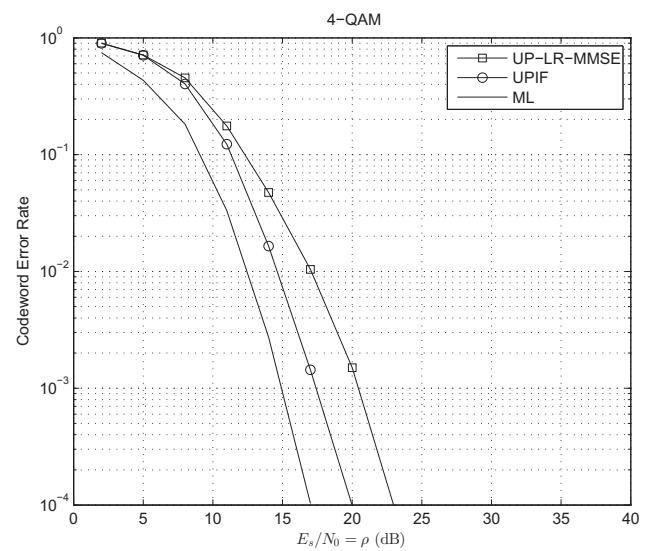

Fig. 3. Unitary Precoded IF in comparison with other schemes in a $4 \times 4$ MIMO Channel. For this figure, both CSIT and precoding are available. The corresponding precoder matrix $\mathbf{P}_{4}$ is given in (19).

Since the performance of UPIF decreases by growing $\rho$ parallel to ML decoded curve in both the figures, full-diversity of UPIF is guaranteed.

\section{Summary And Directions for Future Work}

A unitary precoding scheme has been introduced to be employed at the transmitter of a flat-fading MIMO channel in the presence of both CSIT and CSIR. At the receiver side, an IF linear receiver is employed. This precoding scheme 


$$
\mathbf{P}_{4}=\left(\begin{array}{cccc}
-0.3663925121 & -0.7677000238 & 0.4230815704 & 0.3120820187 \\
-0.2264430248 & -0.4744647078 & -0.6845603618 & -0.5049593142 \\
-0.4744647080 & 0.2264430248 & -0.5049593144 & 0.6845603618 \\
-0.7677000246 & 0.3663925106 & 0.3120820189 & -0.4230815707
\end{array}\right)
$$

followed by a linear receiver is called unitary precoded integerforcing (UPIF). The diversity gains of the proposed approach has been analyzed both theoretically and numerically.

The recently introduced X- and Y-precoders [28] for MIMO channels can be considered as potential competitors to this work as they share three common properties with UPIF. First, they have unitary precoders at the transmitter. Second, they both have low complexity in comparison with joint ML decoders when the number of transmit and receive antennas are small. Third, both achieve full (transmit and receive) diversity in $2 \times 2$ MIMO channels. Comparing the error performance of the proposed UPIF in this work with $\mathrm{X}$ - and Y-precoding schemes is left for the future work.

As this work is a consequence of [29], designing fulldiversity unitary precoders with IF receiver at the destination without having CSIT is of interest. Another direction is to let the transmitter have access to limited feedback over a delayfree link from the IF receiver. In this case, designing a suitable codebook of unitary precoding matrices which attains higher rates and obtain higher coding gains seems to be a promising research topic.

\section{REFERENCES}

[1] E. Viterbo and J.J. Boutros, "A Universal Lattice Decoder for Fading Channels," IEEE Trans. on Inform. Theory, vol. 45, no. 5, pp. 16391642, July. 1999.

[2] K. Kumar, G. Caire, and A. Moustakas, "Asymptotic performance of linear receivers in MIMO fading channels," IEEE Trans. on Inform. Theory, vol. 55, no. 10, pp. 4398-4418, Oct. 2009.

[3] J. Zhan, B. Nazer, U. Erez, and M. Gastpar, "Integer-forcing linear receivers," To appear in IEEE Trans. on Inform. Theory. Available online at arXiv:1003.5966v3.

[4] A. Sakzad, J. Harshan, and E. Viterbo, "Integer-forcing MIMO linear receivers based on lattice reduction," IEEE Trans. on Wireless Communications, vol. 12, no. 10, pp. 4905-4915, Nov. 2013.

[5] A. Sakzad, J. Harshan, and E. Viterbo, "On complex LLL algorithm for integer forcing linear receivers," in the Proc. of in Proc. IEEE Australian Communication Theory Workshop 2013, Adelaide, Australia, Jan. 2013, pp. $13-18$.

[6] V. Tarokh, N. Seshadri, and A.R. Calderbank, "Space-time codes for high data rate wireless communication: performance criterion and code construction," IEEE Trans. on Inform. Theory, vol. 44, no. 2, pp. 744 765, Mar. 1998.

[7] B.A. Sethuraman, B.S. Rajan, and V. Shashidhar, "Full-diversity, highrate space-time block codes from division algebras," IEEE Trans. on Inform. Theory, vol. 49, no. 10, pp. 2596-2616, Oct. 2003.

[8] J.K. Zhang, J. Liu, and K.M. Wong, "Linear Toeplitz space time block codes," in Proc. of IEEE ISIT-05, Adelaide, Australia, Sep. 2005 pp. 1942-1946.

[9] J. Liu, J.K. Zhang, and K.M. Wong, "On the design of minimum BER linear space-time block codes for MIMO systems equipped with MMSE receivers," IEEE Trans. Signal Processing, vol. 54, no. 8, pp. 3147-3158, Aug. 2006.

[10] G. Susinder Rajan and B.S. Rajan, "MMSE Optimal Algebraic SpaceTime Codes," IEEE Trans. on Wireless Communications, vol. 07, no. 7 , pp. 2468-2472, Jul. 2008.
[11] Y. Shang and X.-G. Xia, "Space time block codes achieving full diversity with linear receivers," IEEE Trans. on Inform. Theory, vol. 54, no. 10 pp. 4528-4547, Oct. 2008

[12] W. Zhang, T. Xu, and X.-G. Xia, "Two designs of space-time block codes achieving full diversity with partial interference cancellation group decoding," IEEE Trans. on Inform. Theory, vol. 58, no. 2, pp. 747-764, Oct. 2012.

[13] H. Wang, X.-G. Xia, Q. Yin, and B. Li, "A family of space-time block codes achieving full diversity with linear receivers," IEEE Trans. on Communications, vol. 57, no. 12, pp. 3607-3617, Dec. 2009

[14] C. Peel, B. Hochwald, and A. Swindlehurst, "A vector-perturbation technique for near-capacity multiantenna multiuser communication-part I: channel inversion and regularization," IEEE Trans. Commun., vol. 53, no. 1, pp. 195-202, Jan. 2005.

[15] A.H. Mehana and A. Nosratinia, "Diversity of MIMO Linear Precoding," IEEE Trans. on Inform. Theory, vol. 60, no. 2, pp. 1019-1038, Feb. 2014

[16] O. Ordentlich and U. Erez, "Precoded integer-forcing universally achieves the MIMO capacity to within a constant gap," submitted to IEEE Trans. on Inform. Theory, 2013. Also available online at arXiv:1301.6393v2.

[17] F. Oggier, F.G. Rekaya, J.-C. Belfiore, and E. Viterbo, "Perfect SpaceTime Block Codes," IEEE Trans. on Inform. Theory, vol. 52, no. 9, pp. 3885-3902, Sept. 2006.

[18] S. Bergman and B. Ottersten, "Lattice-based linear pre-coding for MIMO channels with transmitter CSI," IEEE Trans. on Signal Processing, vol. 56, no. 7, pp. 29002-2914, July 2008.

[19] A. Lozano, A. Tulino, and S. Verdu, "Optimum power allocation for parallel Gaussian channels with arbitrary in-put distributions," IEEE Trans. on Inform. Theory, vol. 52 no. 7, pp. 3033-3051, July 2006.

[20] F. Perez-Cruz, M.R. Rodrigues, and S. Verdu, "MIMO Gaussian channels with arbitrary inputs: Optimal precoding and power allocation," IEEE Trans. on Inform. Theory, vol. 56, no. 3, pp. 1070-1084, Mar. 2010.

[21] E. Telatar, "Capacity of Multi-antenna Gaussian Channels," European Trans. on Telecommunications, vol. 10 no. 6, pp. 585-595, 1999

[22] M. Payaro and D.P. Palomar, "Hessian and Concavity of Mutual Information, Differential Entropy, and Entropy Power in Linear Vector Gaussian Channels," IEEE Trans. on Inform. Theory, vol. 55, no. 8, pp. 3613-3628, Aug. 2009.

[23] J.J. Boutros and E. Viterbo "Signal space diversity: a power- and bandwidth-efficient diversity technique for the Rayleigh fading channel," IEEE Trans. on Inform. Theory, vol. 44, no. 4, pp. 1453-1467, July. 1998

[24] List of full diversity algebraic rotations, available online at: http://www.ecse.monash.edu.au/staff/eviterbo/rotations/rotations.html.

[25] J. Conway and N. Sloane, "A fast encoding method for lattice codes and quantizers," IEEE Trans. on Inform. Theory, vol. 29, no. 6, pp. 820-824, Nov. 1983.

[26] M.A. Woodbury, "Inverting modified matrices," Statistical Research Group, Memo. Rep., Princeton University, Princeton, N. J., no. 42 pp. $65.0 \mathrm{X}, 1950$.

[27] C. Feng, D. Silva, and F.R. Kschichang, "An algebraic approach to physical-layer network coding," IEEE Trans. on Inform. Theory, vol., 59 , no. 11, pp. 7576-7596, Nov. 2013.

[28] S.K. Mohammed, E. Viterbo, Y. Hong, and A. Chockalingam, "MIMO Precoding With $\mathrm{X}$ - and Y-Codes," IEEE Trans. on Inform. Theory, vol. 57, no. 6, pp. 3542-3566, June 2011.

[29] A. Sakzad, E. Viterbo, J.J. Boutros, and Y. Hong, "Phase Precoding for the Compute-and-Forward Protocol," submitted for possible publication Available online at: http://arxiv.org/abs/1404.4157. 